\title{
Spectral element analysis of free - vibration of Timoshenko beam
}

\author{
Saida Hamioud(1), Salah Khalfallah(2) \\ (1) Department of Civil Engineering, University of Jijel, Jijel, ALGERIA \\ e-mail: hamioud.saida@yahoo.com \\ (2) Department of Mechanical Engineering, Polytechnic National School, Constantine, ALGERIA \\ e-mail: khalfallah_s25@yahoo.com
}

\section{SUMMARY}

The paper describes free vibration of Timoshenko beam by using spectral element method. Based on the partial differential equation of motion, the dynamic stiffness matrix in the frequency domain is formulated. In this case, natural frequencies for simply supported beam are obtained for each mode of vibration. In this study, three solutions are presented: (1) the analytical solution, (2) the finite element method, and (3) the spectral element method. In the last method, the beam is described by one element only, but in finite element modelling, the number of elements varies in order to improve the quality of the solution. Numerical results obtained by these three methods are collected. The spectral element method displays high performance compared to the finite element approach, and is considered as interesting tool in the structural dynamic field.

KEY WORDS: analytical solution; Euler-Bernoulli beam; finite element method; free vibration; spectral element method; spectral stiffness matrix; Timoshenko beam.

\section{INTRODUCTION}

Timoshenko beam theory is one of the classical models. It was invented in 1921 and developed in 1922 by Timoshenko. Since this year, it has been a topic of various studies in vibration analysis of beam-like structures [1-5]. Until now, the Timoshenko beam theory has already been used to analyse vibration of micro and nano-structures $[2,6]$.

Firstly, the Euler-Bernoulli theory applied for bending beams disregards the effect of shear deformations. This theory is suitable for slender beams and not for thick or deep ones because the transverse shear strains remain null. Since this theory neglects the transverse shear deformations, it underestimates deflections and overestimates the natural frequencies in case of thick beams, where shear deformation effects are significant.

The theory of thick beams was extended by Timoshenko so as to take shear deformations into account. This effect is very strong in higher vibration modes. The Timoshenko beam theory deals with two differential equations of motion in terms of deflection and cross-section rotation. The Timoshenko beam theory has come into focus with considerable developments of the finite element method and its application in practice [7-9]. 
The finite element method (FEM) and the spectral finite element method (SFEM) are among numerical methods used in various computing of static and dynamic responses of structures. In FEM approach, the shape functions are independent of the vibrating frequencies and improved results can be obtained with higher number of elements and degrees of freedom. In contrast, the SFEM reaches accurate solutions with a few numbers of elements and degrees of freedom.

In this context, the finite element method is largely used to analyse free and forced vibration of structures [10-11]. In the other part, Lee and Schultz [12] study free -vibration of Timoshenko beams and axi-symmetric Mindlin plates using the pseudo-spectral method. The finite element analysis vibration of rotating Timoshenko beams is presented by Rao and Gupta [13]. In addition, Katz et al. [14] studied the dynamic behaviour of a rotating shaft subjected to a moving load with constant velocity coupling the modal analysis method and an integral transformation method.

Later, Doyle [15] introduced Fourier transform approach to resolve the governing differential equation of the spectral Timoshenko beam. It has been used to analyse the dynamic of the continuous beam and bridge subjected to a moving load [16]. Thus, this approach is extended to study the dynamic of the cracked Timoshenko beam [17]. In this field, Song et al. [18] studied the vibration of a beam subjected to a moving force in the frequency-domain. Kumar et al. [3] used the spectral element method for wave propagation and structural diagnostic analysis of a composite beam with transverse crack.

In this paper, the formulation using SFEM is devoted to study the vibration of Timoshenko beam. By using the concept of the dynamic spectral method, circular frequencies and mode shapes of vibration are computed, and a parametric study is established. The results of SFEM with respect to FEM display less discretisation of the structure with greater numerical accuracy.

\section{MATHEMATICAL FORMULATIONS}

This section describes the formulation of the simply supported Timoshenko beam vibration using the finite element method, the spectral element method and the mathematical solution.

\subsection{FINITE ELEMENT METHOD}

The mechanical and geometrical characteristics of a prismatic beam are as follow: length $L$, area $\Omega$, moment of inertia $I$, Young's modulus $E$, shear modulus $G$ and the density $\rho$ (Figure 1 ). The Timoshenko theory accounts for an average transverse shear deformation $\gamma$ through thickness. It also includes rotatory inertia of the cross section but the latter has only a minor effect. The effect of transverse shear is much greater than that of rotatory inertia on the response of transverse vibration of prismatic bars. In this theory, transverse shear strain distribution is assumed to be constant through the beam thickness and thus requires the introduction of the shear correction factor $K$ to represent the strain energy of deformation [19]. The shear deformation is related to bending slope $\partial v(x, t) / \partial x$ by:

$$
\frac{\partial v(x, t)}{\partial x}=\theta(x, t)+\gamma(x, t)
$$

$v$ is the transverse displacement of the beam and $\theta$ is the cross section rotation. 

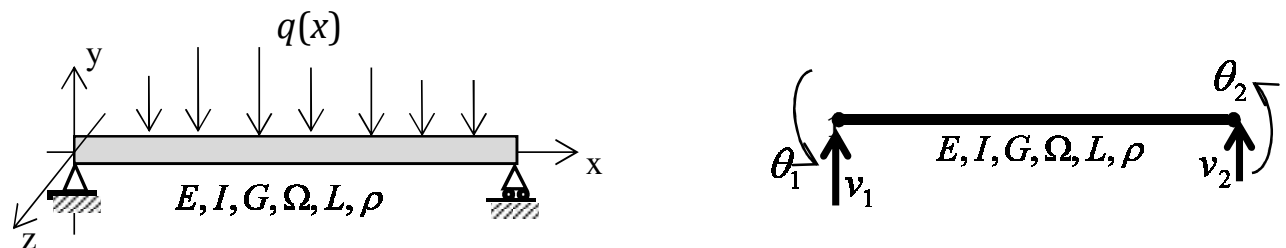

Fig. 1 Geometrical and mechanical characteristics of the used beam

The expression of strain energy $U_{e}$ and the kinetic energy $T_{e}$ of bending and shear effects can be evaluated, respectively:

$$
\begin{gathered}
U_{e}=\frac{1}{2} \int_{L} E I\left(\frac{\partial \theta(x, t)}{\partial x}\right)^{2} d x+\frac{1}{2} \int_{L} K G \Omega\left(\frac{\partial v(x, t)}{\partial x}-\theta(x, t)\right)^{2} d x \\
T_{e}=\frac{1}{2} \int_{0}^{L} \rho \Omega\left(\frac{\partial v^{2}(x, t)}{\partial x^{2}}\right)^{2} d x+\frac{1}{2} \int_{0}^{L} \rho I\left(\frac{\partial \theta(x, t)}{\partial x}\right)^{2} d x
\end{gathered}
$$

By using interpolation functions of the displacement field and those of the shear deformation, the relationship (2) can be expressed as:

$$
\begin{aligned}
& U_{e}=\frac{1}{2}\left\{q_{e}\right\}^{T} \int_{0}^{L} E I\left(\left[\frac{d N_{\theta}(x)}{d x}\right]^{T} \cdot\left[\frac{d N_{\theta}(x)}{d x}\right]\right)\left\{q_{e}\right\} d x+ \\
& +\frac{1}{2}\left\{q_{e}\right\}^{T} \int_{0}^{L} K G \Omega\left(\left[\frac{d N_{v}(x)}{d x}\right]-\left[N_{\theta}(x)\right]\right)^{T}\left(\left[\frac{d N_{v}(x)}{d x}\right]-\left[N_{\theta}(x)\right]\right)\left\{q_{e}\right\} d x
\end{aligned}
$$

The strain energy expression Eq. (4) leads to the formulation of the stiffness matrix components of Timoshenko beam.

$$
\begin{gathered}
{\left[K_{b}\right]=\int_{0}^{L} E I\left[\frac{d N_{\theta}(x)}{d x}\right]^{T}\left[\frac{d N_{\theta}(x)}{d x}\right] d x} \\
{\left[K_{S}\right]=\int_{0}^{L} G \Omega K\left[\frac{d N_{v}(x)}{d x}-N_{\theta}(x)\right]^{T}\left[\frac{d N_{v}(x)}{d x}-N_{\theta}(x)\right] d x}
\end{gathered}
$$

The above process can be applied to evaluate the components of the mass matrix, as:

$$
\begin{aligned}
& {\left[M_{v}\right]=\int_{0}^{L} \rho \Omega\left[N_{v}(x)\right]^{T}\left[N_{v}(x)\right] d x} \\
& {\left[M_{\theta}\right]=\int_{0}^{L} \rho I\left[N_{\theta}(x)\right]^{T}\left[N_{\theta}(x)\right] d x}
\end{aligned}
$$

In this case, integrating boundary conditions in Lagrange's equation can be applied for a simply supported beam, which can lead to the formulation of the equation of free -vibration (the formulations of stiffness and mass matrices are described in Appendix 1 and 2, respectively). The trivial solution of free -vibration of beams is obtained unless.

$$
\left|\left[K_{e}\right]-\omega^{2}\left[M_{e}\right]\right|=0
$$

where $\left[K_{e}\right]=\left[K_{b}\right]+\left[K_{s}\right]$ and $\left[K_{e}\right]=\left[K_{v}\right]+\left[K_{\theta}\right]$. 
In order to compute natural frequencies of the clamped-free beam, two cases can be considered based on the mass hypothesis.

Case 1: Consistent mass hypothesis

The solutions of the Eq. (8) are:

$$
\begin{gathered}
\omega_{c 1}=\frac{2 \sqrt{30}}{\rho \Omega L} \sqrt{\frac{\rho \Omega E I}{L^{2}+10 r^{2}}} \\
\omega_{c 2}=\frac{6 \sqrt{70}}{\rho \Omega L} \sqrt{\frac{E I \rho \Omega(\lambda+1)}{42 r^{2}\left(5 \lambda^{2}+1\right)+L^{2}}}
\end{gathered}
$$

with $r=\sqrt{I / \Omega}$ and $\lambda=12 E I /\left(K G \Omega L^{2}\right)$.

Case 2: Lumped mass hypothesis

In this case, the solution of Eq. (8) is:

$$
\omega_{l 1}=\frac{1}{L(1+\lambda)} \sqrt{\frac{2 E I}{\rho \Omega} \frac{16+40 \lambda+33 \lambda^{2}+10 \lambda^{3}+\lambda^{4}}{4+5 \lambda+\lambda^{2}}}
$$

By comparing natural frequencies (9) and (10), the consistent mass hypothesis is required for the analysis presenting rotational pulsations of the beam (9).

\subsection{ANALYTICAL METHOD}

\subsubsection{EQUATION OF MOTION}

The Figure 2 shows a free body diagram of an extracted element from Timoshenko beam where $M(x, t)$ is the bending moment, $T(x, t)$ is the shear force, $\rho \Omega\left(\partial^{2} v(x, t) / d t^{2}\right) d x$ is the inertia force, $\theta(x, t)$ is the slope of the beam due to bending, $\rho I\left(\partial^{2} \theta(x, t) / \partial t^{2}\right) d x$ is rotatory inertia, and $q(x)$ is the applied loading.

The equilibrium equation according to the $z$-axis results in:

$$
\frac{\partial T(x, t)}{\partial x}+\rho \Omega \frac{\partial^{2} v(x, t)}{\partial t^{2}}+q(x)=0
$$

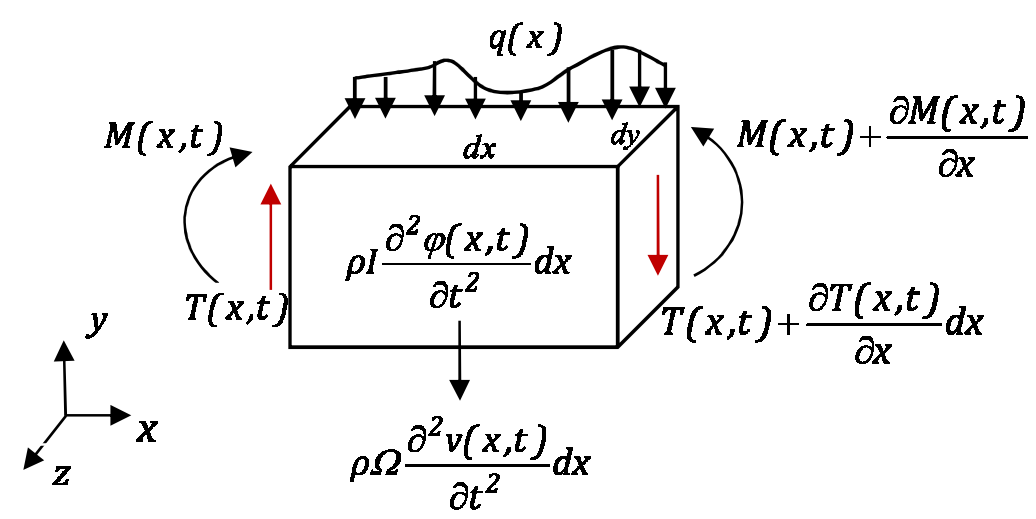

Fig. 2 Free-body diagram of an element of length $d x$ 
For the free -vibration approach, external loads are neglected. The Eq. (11) can be written in general partial differential equation as:

$$
K \Omega G\left(\frac{\partial^{2} v(x, t)}{\partial x^{2}}-\frac{\partial \theta(x, t)}{\partial x}\right)-\rho \Omega \frac{\partial^{2} v(x, t)}{\partial t^{2}}=0
$$

The balanced bending moment about the centre point of the element is:

$$
\frac{\partial M(x, t)}{\partial x}-\frac{\partial T(x, t)}{\partial x} \frac{d x}{2}-\rho I \frac{\partial^{2} \varphi(x, t)}{\partial t^{2}}=0
$$

The relationship (13) can be written in the following form:

$$
E I \frac{\partial^{2} \theta(x, t)}{\partial x^{2}}+K G \Omega\left(\frac{\partial v(x, t)}{\partial x}-\theta(x, t)\right)-\rho I \frac{\partial^{2} \theta(x, t)}{\partial t^{2}}=0
$$

Using separated variables of $v(x, t)=X(x) e^{i \omega t}$ and $\theta(x, t)=Y(x) e^{i \omega t}$, the system of Eqs. (12) and (14) can be written in the following matrix form:

$$
\begin{aligned}
& {\left[\begin{array}{cc}
K G \Omega & 0 \\
0 & E I
\end{array}\right]\left\{\begin{array}{l}
X^{\prime \prime}(x) \\
Y^{\prime \prime}(x)
\end{array}\right\}+\left[\begin{array}{cc}
0 & -K G \Omega \\
K G \Omega & 0
\end{array}\right]\left\{\begin{array}{l}
X^{\prime}(x) \\
Y^{\prime}(x)
\end{array}\right\}+} \\
& +\left[\begin{array}{cc}
\omega^{2} \rho \Omega & 0 \\
0 & \omega^{2} \rho I-K G \Omega
\end{array}\right]\left\{\begin{array}{l}
X(x) \\
Y(x)
\end{array}\right\}=\left\{\begin{array}{l}
0 \\
0
\end{array}\right\}
\end{aligned}
$$

The functions $X(x)$ and $Y(x)$ are identical, hence, it is possible to put:

$$
X(x)=\frac{Y(x)}{\beta}=C e^{\alpha x}
$$

By substituting Eq. (16) into Eq. (15), we obtain:

$$
\left[\begin{array}{cc}
K G \Omega \alpha^{2}+\omega^{2} \rho \Omega & -K G \Omega \alpha \\
K G \Omega \alpha & E I \alpha^{2}+\omega^{2} \rho I-K G \Omega
\end{array}\right]\left\{\begin{array}{l}
1 \\
\beta
\end{array}\right\}=\left\{\begin{array}{l}
0 \\
0
\end{array}\right\}
$$

In order to obtain non-trivial solutions, the determinant of the above matrix must be zero.

$$
\alpha^{4}+\alpha_{F}^{4} \eta \alpha^{2}+\alpha_{F}^{4}\left(\alpha_{G}^{4} \eta_{1}-1\right)=0
$$

with $\alpha_{F}=\sqrt{\omega}\left(\frac{\rho \Omega}{E I}\right)^{1 / 4}, \alpha_{G}=\sqrt{\omega}\left(\frac{\rho}{k G}\right)^{1 / 4}, \eta_{1}=\frac{I}{\Omega}, \eta_{2}=\frac{E I}{k G \Omega}$ and $\eta=\eta_{1}+\eta_{2}$.

The four roots of the Eq. (18) are as follows:

$$
\begin{gathered}
\alpha_{1}=i \frac{\alpha_{F}}{\sqrt{2}} \sqrt{\alpha_{F}^{2} \eta+\sqrt{\left(\alpha_{F}^{4} \eta^{2}-4\left(\alpha_{G}^{4} \eta_{1}-1\right)\right)}}=i \lambda_{1} \\
\alpha_{2}=-i \lambda_{1} \\
\alpha_{3}=\frac{\alpha_{F}}{\sqrt{2}} \sqrt{-\alpha_{F}^{2} \eta+\sqrt{\left(\alpha_{F}^{4} \eta^{2}-4\left(\alpha_{G}^{4} \eta_{1}-1\right)\right)}}=\lambda_{2} \\
\alpha_{4}=-\lambda_{2}
\end{gathered}
$$

Spatial solutions are then expressed as:

$$
X(x)=A_{1} \cos \left(\lambda_{1} x\right)+A_{2} \sin \left(\lambda_{1} x\right)+A_{3} e^{\lambda_{2} x}+A_{4} e^{-\lambda_{2} x}
$$




$$
Y(x)=\beta_{1} A_{1} \cos \left(\lambda_{1} x\right)+\beta_{2} A_{2} \sin \left(\lambda_{1} x\right)+\beta_{3} A_{3} e^{\lambda_{2} x}+\beta_{4} A_{4} e^{-\lambda_{2} x}
$$

with $\beta_{n}=\alpha_{n}+\frac{\alpha_{G}^{2}}{\alpha_{n}}: n=1,2,3, \ldots$

The application of boundary conditions for the simply supported beam $(X(x=0, \omega)=0$, $X(x=L, \omega)=0, X^{\prime \prime}(x=0, \omega)=0$ and $\left.X^{\prime \prime}(x=L, \omega)=0\right)$ results in:

$$
[A(\lambda L)]=\left[\begin{array}{cccc}
1 & 0 & 1 & 1 \\
-1 & 0 & 1 & 1 \\
\cos \left(\lambda_{1} L\right) & \sin \left(\lambda_{1} L\right) & e^{\lambda_{2} L} & e^{-\lambda_{2} L} \\
-\cos \left(\lambda_{1} L\right) & -\sin \left(\lambda_{1} L\right) & e^{\lambda_{2} L} & e^{-\lambda_{2} L}
\end{array}\right]
$$

with $\left\langle X(x=0, \omega), X^{\prime \prime}(x=0, \omega), X(x=L, \omega), X^{\prime \prime}(x=L, \omega)\right\rangle^{t}=[A(\lambda L)]\left\langle A_{1}, A_{2}, A_{3}, A_{4}\right\rangle^{t}$.

For a non-trivial solution of $X(x)$, the determinant of the matrix (21) must be null:

$$
8 \sin \left(\lambda_{1} L\right) \cdot \sinh \left(\lambda_{2} L\right)=0
$$

Corresponding solutions of the Eq. (22) are valid for:

$$
\begin{gathered}
\lambda_{n}=\frac{n \pi}{L} \\
\text { for } \quad n=1,2,3, \ldots . .
\end{gathered}
$$

\subsection{SPECTRAL ELEMENT METHOD}

The matrix differential Eq. (15) can be solved by using the Fourier transform. The solution is considered as the sum of harmonic vibration:

$$
\begin{aligned}
& v(x, t)=\frac{1}{N} \sum_{n=0}^{N-1} W(x, \omega) e^{i \omega_{n} t} \\
& \theta(x, t)=\frac{1}{N} \sum_{n=0}^{N-1} \Phi(x, \omega) e^{i \omega_{n} t}
\end{aligned}
$$

The functions $W(x, \omega)$ and $\Phi(x, \omega)$ and (24) can be expressed as:

$$
\begin{aligned}
& W(x, \omega)=A e^{i \alpha \omega x} \\
& \Phi(x, \omega)=\beta A e^{i \alpha \omega x}
\end{aligned}
$$

By substituting Eq. (25) into Eq. (15), we obtain:

$$
\left[\begin{array}{cc}
\rho \Omega \omega^{2}-K G \Omega \alpha^{2} & -K \Omega G \alpha i \\
K \Omega G \alpha i & \rho I \omega^{2}-K \Omega G-E I \alpha^{2}
\end{array}\right]\left\{\begin{array}{l}
1 \\
\beta
\end{array}\right\}=\left\{\begin{array}{l}
0 \\
0
\end{array}\right\}
$$

Also, for non-trivial solutions, the determinant of the Eq. (26) results in the same Eq. (18) and the corresponding solutions can be obtained by substituting $\lambda_{i}$ with $\alpha_{i}$.

$$
W(x)=\left(A_{1} e^{i \alpha_{1} x}+A_{2} e^{-i \alpha_{1} x}+A_{3} e^{i \alpha_{2} x}+A_{4} e^{-i \alpha_{2} x}\right)
$$




$$
\Phi(x)=\left(\beta_{1} A_{1} e^{i \alpha_{1} x}-\beta_{1} A_{2} e^{-i \alpha_{1} x}+\beta_{2} A_{3} e^{i \alpha_{2} x}-\beta_{2} A_{4} e^{-i \alpha_{2} x}\right)
$$

with $\beta_{1}=\frac{i}{\alpha_{1}}\left(\alpha_{1}^{2}-\alpha_{G}^{2}\right)$ and $\beta_{2}=\frac{i}{\alpha_{2}}\left(\alpha_{2}^{2}-\alpha_{G}^{2}\right)$.

Nodal displacements at the ends of the free-body beam element can be deduced as follows:

$$
\begin{aligned}
\left\{q_{e}\right\}=\left\{\begin{array}{l}
v_{1} \\
\theta_{1} \\
v_{2} \\
\theta_{2}
\end{array}\right\}=\left[\begin{array}{cccc}
1 & 1 & 1 & 1 \\
\beta_{1} & -\beta_{1} & \beta_{2} & -\beta_{2} \\
e_{1} & e_{1}^{-1} & e_{2} & e_{2}^{-1} \\
\beta_{1} e_{1} & -\beta_{1} e_{1}^{-1} & \beta_{2} e_{2} & -\beta_{2} e_{2}^{-1}
\end{array}\right]\left\{\begin{array}{l}
A_{1} \\
A_{2} \\
A_{3} \\
A_{4}
\end{array}\right\} \\
e_{1}=e^{i \alpha_{1} L} \text { and } e_{2}=e^{i \alpha_{2} L}
\end{aligned}
$$

In compact form, the relation (28) can be expressed as:

$$
\left\{q_{e}\right\}=[D(\omega)]\{A\}
$$

Equivalent loads at the beam element ends can be deduced as follows:

$$
\left\{\begin{array}{l}
T_{1} \\
M_{1} \\
T_{2} \\
M_{2}
\end{array}\right\}=\left[\begin{array}{cccc}
K \Omega G C_{1} & -K \Omega G C_{1} & K \Omega G C_{2} & -K \Omega G C_{2} \\
-i E I r_{1} & -i E I r_{1} & -i E I r_{2} & -i E I r_{2} \\
-K \Omega G C_{1} e_{1} & K \Omega G C_{1} e_{1}^{-1} & -K \Omega G C_{2} e_{2} & K \Omega G C_{2} e_{2}^{-1} \\
i E I r_{1} e_{1} & i E I r_{1} e_{1}^{-1} & i E I r_{2} e_{2} & i E I r_{4} e_{2}^{-1}
\end{array}\right] \cdot\left\{\begin{array}{l}
A_{1} \\
A_{2} \\
A_{3} \\
A_{4}
\end{array}\right\}
$$

with $C_{i}=\beta_{i}-i \alpha_{i}: i=1,2$ and $r_{j}=\alpha_{j} \beta_{j}: j=1,2$.

Substituting Eq. (29) into Eq. (30), the relation between the load vector and the displacement vector is:

$$
\begin{gathered}
\left\{F_{e}\right\}=[F(\omega)][D(\omega)]^{-1}\left\{q_{e}\right\} \\
\text { where }\left\{F_{e}\right\}=\left\langle T_{1}, M_{1}, T_{2}, M_{2}\right\rangle^{t}
\end{gathered}
$$

Thus, the matrix $[F(\omega)] \cdot[D(\omega)]^{-1}$ describes the spectral stiffness matrix of Timoshenko beam (the parameters of the spectral stiffness matrix are regrouped in Appendix 3).

In order to obtain natural frequencies of free -vibration of the beam, the determinant of spectral element matrix must be null.

$$
\cos \left(\alpha_{2} L\right)+i \sin \left(\alpha_{2} L\right)=-1
$$

The corresponding solutions of Eq. (32) are:

$$
\begin{gathered}
\alpha_{2}=\frac{(2 n+1) \pi}{L} \\
\text { for } n=1,2,3, \ldots
\end{gathered}
$$




\section{RESULTS}

In this section, a simply supported beam (Figure 3) is studied and the above approach can easily be applied to other different boundary conditions.

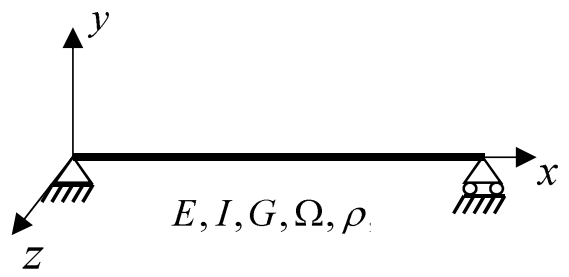

Fig. 3 Studied beam

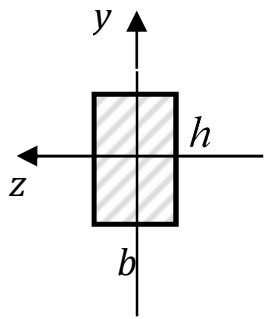

\subsection{FREE -VIBRATION RESPONSE}

Mechanical and geometrical properties of the rectangular steel beam are regrouped in Table 1. SFEM, FEM numerical and exact solutions are regrouped in Table (2), simultaneously. In FEM approach, the beam is discretised with various meshes as: 4, 8, 15, 20, 50 and 100 elements.

Table 1 Properties of the studied beam

\begin{tabular}{|c|c|c|c|c|c|c|c|}
\hline$L(m)$ & $b(m)$ & $h(m)$ & $v$ & $E(M P a)$ & $G(M P a)$ & $\rho\left(N / m^{3}\right)$ & $k$ \\
\hline 1 & 0.05 & 0.15 & 0.305 & $207 \cdot 10^{9}$ & $79.3 \cdot 10^{9}$ & $76.5 \cdot 10^{3}$ & $5 / 6$ \\
\hline
\end{tabular}

Table 2 illustrates the first six natural frequencies computed using SFEM, FEM and mathematical method. For first modes of vibration, the difference between numerical and exact solutions is not notable and not valid for higher modes of vibration. This difference becomes very important from the third mode of vibration. In this case, natural frequencies using numerical method are rather far from the real ones that take into account superior modes of vibration in the structure design.

Table 2 First six frequencies using different methods (rad/s)

\begin{tabular}{|c|c|c|c|c|c|c|c|}
\hline mode & $\mathbf{1}$ & $\mathbf{2}$ & $\mathbf{3}$ & $\mathbf{4}$ & $\mathbf{5}$ & $\mathbf{6}$ & $\begin{array}{c}\text { Ratio } \\
\mathbf{\%}\end{array}$ \\
\hline $\mathbf{4} \mathbf{F E}$ & 678.9626 & 2523.1884 & 5317.5238 & 10949.4933 & 15360.8339 & 21538.3718 & 55.45 \\
\hline $\boldsymbol{8} \mathbf{F E}$ & 678.1246 & 2484.7364 & 5037.1170 & 8115.9613 & 11660.0463 & 15630.1474 & 12.81 \\
\hline $\mathbf{1 5} \mathbf{F E}$ & 677.9497 & 2476.4928 & 4972.8335 & 7871.7748 & 11030.1274 & 14394.4763 & 3.89 \\
\hline $\mathbf{2 0} \mathbf{F E}$ & 677.9203 & 2475.0985 & 4961.8901 & 7829.7686 & 10918.8727 & 14158.6384 & 2.19 \\
\hline $\mathbf{4 0} \mathbf{F E}$ & 677.8922 & 2473.7651 & 4951.4092 & 7789.4797 & 10811.9601 & 13931.1727 & 0.55 \\
\hline $\mathbf{5 0} \mathbf{F E}$ & 677.8889 & 2473.6058 & 4950.1563 & 7784.6606 & 10799.1660 & 13903.9366 & 0.35 \\
\hline $\mathbf{1 0 0} \mathbf{F E}$ & 677.8844 & 2473.3937 & 4948.4875 & 7778.2407 & 10782.1210 & 13867.6529 & 0.09 \\
\hline $\mathbf{1 S E}$ & $\mathbf{6 7 7 . 8 8 2 9}$ & $\mathbf{2 4 7 3 . 3 2 3 1}$ & $\mathbf{4 9 4 7 . 9 3 1 6}$ & $\mathbf{7 7 7 6 . 1 0 2 1}$ & $\mathbf{1 0 7 7 6 . 4 4 3 0}$ & $\mathbf{1 3 8 5 5 . 5 6 6 9}$ & $\mathbf{0 . 0 0}$ \\
\hline Exact & $\mathbf{6 7 7 . 8 8 2 9}$ & $\mathbf{2 4 7 3 . 3 2 3 1}$ & $\mathbf{4 9 4 7 . 9 3 1 6}$ & $\mathbf{7 7 7 6 . 1 0 2 1}$ & $\mathbf{1 0 7 7 6 . 4 4 3 0}$ & $\mathbf{1 3 8 5 5 . 5 6 6 9}$ & $\mathbf{0 . 0 0}$ \\
\hline
\end{tabular}


Additionally, the accuracy of FEM results depends on the beam meshes. In this case, it is recommended to use more than 100 finite elements to reach improved results. On the other side, only one element of spectral element method is sufficient to achieve accurate results.

\subsection{EULER-BERNOULLI AND TIMOSHENKO BEAMS}

Based on the obtained natural frequencies, Table 3 shows a comparison between EulerBernoulli and Timoshenko beams. The effect of shear deformations becomes even more important as the frequencies increase for $h / L=0.15$.

Table 3 Natural frequencies of Euler-Bernoulli and Timoshenko beams for $h / L=0.15$

\begin{tabular}{|c|c|c|c|}
\hline $\begin{array}{c}\text { Mode of } \\
\text { vibration }\end{array}$ & $\begin{array}{c}\text { Euler-Bernoulli beam } \\
(\mathrm{rad} / \mathrm{s})\end{array}$ & $\begin{array}{c}\text { Timoshenko beam } \\
(\mathrm{rad} / \mathrm{s})\end{array}$ & Ratio (\%) \\
\hline 1 & 702.9992 & 677.8829 & 3.70 \\
\hline 2 & 2811.9968 & 2473.3231 & 13.69 \\
\hline 3 & 6326.9929 & 4947.9316 & 27.87 \\
\hline 4 & 11247.9873 & 7776.1021 & 44.64 \\
\hline 5 & 17574.9802 & 10776.4430 & 63.09 \\
\hline 6 & 25307.9715 & 13855.5669 & 82.65 \\
\hline
\end{tabular}

Moreover, for low-frequency range, a relative agreement between dynamic stiffness of Timoshenko beam and Euler-Bernoulli beam can be observed.

In the second case, the beam with different dimensions has been used to explain the slenderness ratio $h / L$ effect. Mechanical and geometrical properties of the rectangular beam are regrouped in Table 4. Table 5 shows the comparison between Euler-Bernoulli and Timoshenko beams for $h / L=0.0066$.

Table 4 Properties of the studied beam

\begin{tabular}{|c|c|c|c|c|c|c|c|}
\hline$L(m)$ & $b(m)$ & $h(m)$ & $v$ & $E(M P a)$ & $G(M P a)$ & $\rho\left(\mathrm{kg} / \mathrm{m}^{3}\right)$ & $k$ \\
\hline 6 & 0.02 & 0.04 & 0.33 & $72.7 \cdot 10^{9}$ & $27.331 \cdot 10^{9}$ & 2700 & $5 / 6$ \\
\hline
\end{tabular}

Tables 3 and 5 show the effect of the slender ratio on the free -vibration of beams. When this ratio is very small, Bernoulli and Timoshenko beams vibrate identically but when the slender ratio of beams becomes significant, Euler-Bernoulli beam vibrates with important natural frequencies. This effect is more pronounced if the higher modes of vibration are taken in consideration. Hence, Euler-Bernoulli beam is stiffer, and the Timoshenko beam works well for short span and thick beams. Therefore, such approach may not be accurate in certain cases when calculating high natural frequencies and eigen-modes. In Euler-Bernoulli beam, the cross section is perpendicular to the bending central line, but in Timoshenko beam, rotation between the cross section and the bending line is considered. This rotation is due to shear deformation, which is not included in Euler-Bernoulli beam. For this reason, Euler-Bernoulli beam is stiffer. 
Table 5 Natural frequencies of Euler-Bernoulli and Timoshenko beams for $h / L=0.0066$

\begin{tabular}{|c|c|c|c|}
\hline Mode & Euler-Bernoulli beam $(\mathrm{rad} / \mathrm{s})$ & Timoshenko beam $(\mathrm{rad} / \mathrm{s})$ & Ratio (\%) \\
\hline 1 & 16.4268 & 16.4255 & 0.008 \\
\hline 2 & 65.7070 & 65.6869 & 0.030 \\
\hline 3 & 147.8408 & 147.7390 & 0.068 \\
\hline 4 & 262.8282 & 262.5067 & 0.122 \\
\hline 5 & 410.6690 & 409.8852 & 0.191 \\
\hline 6 & 591.3634 & 589.7406 & 0.275 \\
\hline
\end{tabular}

\subsection{INFLUENCE OF THE CROSS SECTION SHAPE}

In this section, two different cross sections are used: (1) a circular cross-section and (2) a rectangular cross -section of same surface. Natural frequencies of studied cases are regrouped in Table 6. The rectangular section of beams presents a performance opposite to the circular section. This performance is approximately $62.72 \%$ for the first three modes of vibration, and around $36 \%$ for the other three modes of vibration. It can be discerned from Table 6 that the performance of the cross section nature decreases in correspondence to the higher mode of vibration.

Table 6 Natural frequencies of geometrical cross -sections

\begin{tabular}{|c|c|c|c|}
\hline Mode & Circular section & Rectangular section & Ratio \% \\
\hline $\mathbf{1}$ & $\mathbf{3 9 2 . 0 0 1 7}$ & $\mathbf{6 7 7 . 8 8 2 9}$ & $\mathbf{7 2 . 9 3}$ \\
\hline 2 & 1516.9685 & 2473.3231 & 63.04 \\
\hline 3 & 3248.6378 & 4947.9316 & 52.31 \\
\hline 4 & 5438.8499 & 7776.1021 & 42.97 \\
\hline 5 & 7956.1744 & 10776.4430 & 35.45 \\
\hline 6 & 10699.2925 & 13855.5669 & 29.50 \\
\hline
\end{tabular}

\subsection{INFLUENCE OF THE MATERIAL PROPERTIES}

Table 7 presents natural frequencies for different material properties. In this concept, three values of Young's modulus (three materials) are selected: $E=207 \cdot 10^{3} \mathrm{GPa}, 3 E / 4$ and $E / 2$. Obtained results demonstrated that mechanical nature of the used material influences the dynamic response of the beams. In this case, an evident conclusion can be drawn; as better material quality of the structure is used, free -vibration occurs.

Table 7 Natural frequencies of different Young's modulus values

\begin{tabular}{|c|c|c|c|}
\hline Mode & $E$ & $3 E / 4$ & $E / 2$ \\
\hline 1 & 677.8829 & 587.0659 & 479.3373 \\
\hline 2 & 2473.3231 & 2141.9845 & 1748.9230 \\
\hline 3 & 4947.9316 & 4285.1172 & 3498.7836 \\
\hline 4 & 7776.1021 & 6734.4803 & 5498.6801 \\
\hline 5 & 10776.4430 & 9332.9761 & 7620.3430 \\
\hline 6 & 13855.5669 & 11999.7206 & 9797.7308 \\
\hline
\end{tabular}




\subsection{VIBRATION MODES}

Free -vibration mode shapes of Timoshenko beam for the first five modes are illustrated in Figures (4-5) showing transversal and rotational shape modes of the beam vibration, respectively. In the previous section, it has been demonstrated that Timoshenko beam is sensitive to slenderness ratio. In the previous section, two slenderness ratios have been considered for numerical results. The finite element method is used to obtain mode shapes of vibration for the first five modes of rotation of Timoshenko beam. These results are obtained for a slenderness ratio of 0.15 , and a cross-section shape factor of 5/6. The comparison of transversal and rotational vibrations is provided, as well as an excellent agreement between exact solutions and computed results.

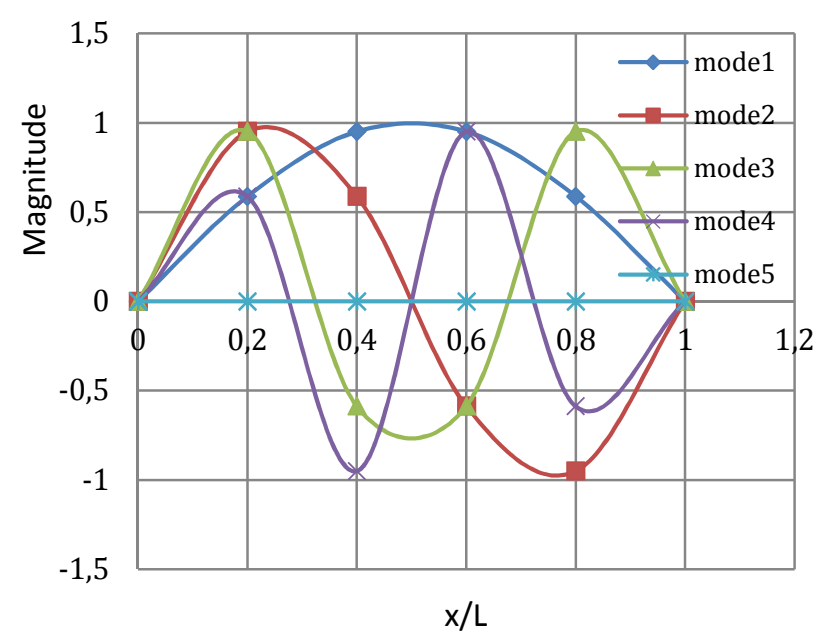

Fig. 4 Transversal vibration of the first five modes

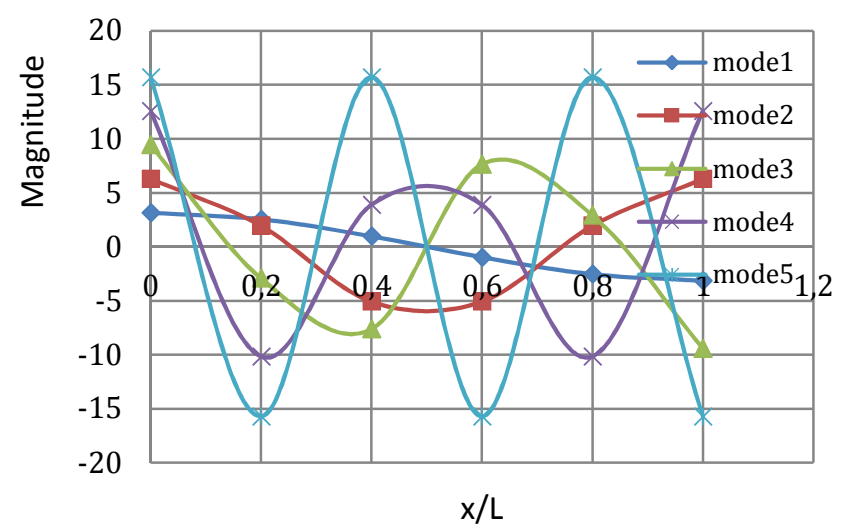

Fig. 5 Rotational vibration of the first five modes 


\section{CONCLUSIONS}

In this paper, SFEM formulation for free -vibration of simply supported Timoshenko beams was examined. The formulation of the dynamic stiffness method has been established, which resulted in Timoshenko beam responses. The accuracy of obtained results by SFEM displays the performance of this approach as compared to FEM. The following conclusions can be draw:

- The spectral element method requires few elements to describe the dynamic beam response.

- In order to reach exact solutions, FEM requires about 100 linear finite element beams.

- For accurate FEM results, superior modes of vibration must be integrated into the analysis.

- The slender ratio has an effect on the free -vibration of beams. For low-frequency range, a good agreement between the dynamic stiffness of Timoshenko beam and Euler-Bernoulli has been observed. However, they vibrate with significant natural frequencies for considerable slenderness ratio values. This effect became more pronounced when higher modes of vibration were taken into consideration.

- In particular, Timoshenko beam is sensitive to slenderness ratio.

- The rectangular cross-section presents high performance opposite to the circular section. The performance is evaluated to $62.72 \%$. Therefore, the better quality of the mechanical material property caused the response of the free -vibration of the beam.

- In general, SEFM closely follows the exact solution and needs less computational effort.

- This approach can be used to analyse beam response with different boundary conditions.

\section{APPENDIX}

\section{Appendix 1: Stiffness matrix of the beam}

The transverse displacement function $v(x)$ of a beam is:

$$
v(x)=a_{1} x^{3}+a_{2} x^{2}+a_{3} x+a_{4}
$$

The derivative of the displacement field of the beam can be deduced:

$$
\theta(x)=a_{2}+2 a_{3} x+a_{4}\left(3 x^{2}+\frac{6 E I}{K G \Omega}\right)
$$

The transversal displacement $v(x)$ and the slope $\theta(x)$ can be expressed in terms of the nodal displacement vector $\left\{q_{e}\right\}=\left\langle v_{1} \theta_{1} v_{2} \theta_{2}\right\rangle^{t}$ :

$$
\begin{aligned}
& v(x)=\left[N_{v}(x)\right]^{T}\left\{q_{e}\right\} \\
& \theta(x)=\left[N_{\theta}(x)\right]^{T}\left\{q_{e}\right\}
\end{aligned}
$$

where: 


$$
\left[N_{v}(x)\right]=\frac{1}{1+\lambda}\left[\begin{array}{l}
1-3 \varepsilon^{2}+2 \varepsilon^{3}+(1-\varepsilon) \lambda \\
\left(\varepsilon-2 \varepsilon^{2}+\varepsilon^{3}+\left(\varepsilon-\varepsilon^{2}\right) \frac{\lambda}{2}\right) L \\
3 \varepsilon^{2}-2 \varepsilon^{3}+\varepsilon \lambda \\
\left(-\varepsilon^{2}+\varepsilon^{3}-\left(\varepsilon-\varepsilon^{2}\right) \frac{\lambda}{2}\right) L
\end{array}\right]
$$

and

$$
\left[N_{\theta}(x)\right]=\frac{1}{(1+\lambda)}\left[\begin{array}{l}
\frac{6}{L}\left(\varepsilon^{2}-\varepsilon\right) \\
1-4 \varepsilon+3 \varepsilon^{2}+(1-\varepsilon) \lambda \\
\frac{6}{L}\left(\varepsilon-\varepsilon^{2}\right) \\
-2 \varepsilon+3 \varepsilon^{2}+\varepsilon \lambda
\end{array}\right]
$$

with $\varepsilon=\frac{X}{L}$.

The stiffness matrix due to the bending effect is:

$$
\left[K_{b}\right]=\frac{E I}{L^{3}(1+\lambda)^{2}}\left[\begin{array}{cccc}
12 & 6 L & -12 & 6 L \\
& \left(4+2 \lambda+\lambda^{2}\right) L^{2} & -6 L & \left(2-2 \lambda-\lambda^{2}\right) L^{2} \\
& & 12 & -6 L \\
\text { sym } & & & \left(4+2 \lambda+\lambda^{2}\right) L^{2}
\end{array}\right]
$$

and the corresponding matrix due to shear deformations is:

$$
\left[K_{s}\right]=\frac{K G \Omega \lambda^{2}}{4 L(1+\lambda)^{2}}\left[\begin{array}{cccc}
4 & 2 L & -4 & 2 L \\
& L^{2} & -2 L & L^{2} \\
& & 4 & -2 L \\
\text { sym } & & & L^{2}
\end{array}\right]
$$

\section{Appendix 2: Mass matrix of the beam}

The total mass matrix of Timoshenko beam can be deduced by using the same process of stiffness matrix formulation:

$$
\left[M_{e}\right]=\left[M_{v}\right]+\left[M_{\theta}\right]
$$

where:

$$
\left[M_{v}\right]=\frac{\rho \Omega L}{(1+\lambda)^{2}}\left[\begin{array}{cccc}
m_{1} & m_{2} & m_{3} & m_{4} \\
& m_{5} & -m_{4} & m_{6} \\
& & m_{1} & -m_{2} \\
\text { sym } & & & m_{5}
\end{array}\right]
$$




$$
\left[M_{\theta}\right]=\frac{\rho \Omega L}{(1+\lambda)^{2}}\left(\frac{r}{L}\right)^{2}\left[\begin{array}{cccc}
m_{7} & m_{8} & -m_{7} & m_{8} \\
& m_{9} & -m_{8} & m_{10} \\
& & m_{7} & -m_{8} \\
s y m & & & m_{9}
\end{array}\right]
$$

with:

$$
\begin{gathered}
m_{1}=\frac{13}{35}+\frac{7}{10} \lambda+\frac{1}{3} \lambda^{2}, m_{2}=\left(\frac{11}{210}+\frac{11}{120} \lambda+\frac{1}{24} \lambda^{2}\right) L, m_{3}=\frac{9}{70}+\frac{3}{10} \lambda+\frac{1}{6} \lambda^{2}, \\
m_{4}=-\left(\frac{13}{420}+\frac{3}{40} \lambda+\frac{1}{24} \lambda^{2}\right) L, m_{5}=\left(\frac{1}{105}+\frac{1}{60} \lambda+\frac{1}{120} \lambda^{2}\right) L^{2}, \\
m_{6}=-\left(\frac{1}{140}+\frac{1}{60} \lambda+\frac{1}{120} \lambda^{2}\right) L^{2}, m_{7}=\frac{6}{5}, m_{8}=\left(\frac{1}{10}-\frac{1}{2} \lambda\right) L, \\
m_{9}=\left(\frac{2}{15}+\frac{1}{6} \lambda+\frac{1}{3} \lambda^{2}\right) L^{2}, m_{10}=-\left(\frac{1}{30}+\frac{1}{6} \lambda-\frac{1}{6} \lambda^{2}\right) L^{2}
\end{gathered}
$$

\section{Appendix 3: Spectral stiffness matrix}

The parameters of the spectral stiffness matrix are:

$$
\left[K_{\text {spect }}\right]=\left[\begin{array}{cccc}
K_{11} & K_{12} & K_{13} & K_{14} \\
K_{21} & K_{22} & K_{23} & K_{24} \\
K_{13} & -K_{14} & K_{11} & -K_{12} \\
-K_{23} & K_{24} & -K_{21} & K_{22}
\end{array}\right]
$$

where:

$$
\begin{gathered}
K_{11}=K_{33}=-\eta_{k} K \Omega G\left(\beta_{1} C_{2}-\beta_{2} C_{1}\right)\left[\left(\beta_{1}-\beta_{2}\right)\left(1-e_{1}^{2} e_{2}^{2}\right)+\left(\beta_{1}+\beta_{2}\right)\left(e_{2}^{2}-e_{1}^{2}\right)\right] \\
K_{22}=K_{44}=i \eta_{K} E I\left(r_{1}-r_{2}\right)\left[\left(\beta_{1}-\beta_{2}\right)\left(1-e_{1}^{2} e_{2}^{2}\right)+\left(\beta_{1}+\beta_{2}\right)\left(e_{1}^{2}+e_{2}^{2}\right)\right] \\
K_{12}=-K_{34}=\eta_{K} K \Omega G\left[\left(C_{2}-C_{1}\right)\left(\beta_{1}-\beta_{2}\right)\left(1+e_{1}^{2} e_{2}^{2}\right)+\left(e_{1}^{2}+e_{2}^{2}\right)\left(\beta_{1}+\beta_{2}\right)\left(C_{2}+C_{1}\right)-4 e_{1} e_{2}\left(\beta_{1} C_{2}+\beta_{2} C_{1}\right)\right] \\
K_{13}=K_{31}=2 \eta_{K} K \Omega G\left(\beta_{1} C_{2}-\beta_{2} C_{1}\right)\left[\beta_{1} e_{2}\left(1-e_{1}^{2}\right)+\beta_{2} e_{1}\left(e_{2}^{2}-1\right)\right] \\
K_{14}=-K_{32}=-2 \eta_{K} K \Omega G\left(\beta_{1} C_{2}-\beta_{2} C_{1}\right)\left(e_{1}-e_{2}\right)\left(1-e_{1} e_{2}\right) \\
K_{21}=-i \eta_{K} E I\left[\left(\beta_{1}^{2} r_{2}+\beta_{2}^{2} r_{1}\right)\left(e_{1}^{2}+e_{2}^{2}-1-e_{1}^{2} e_{2}^{2}\right)+\beta_{1} \beta_{2}\left(r_{1}+r_{2}\right)\left(1+e_{1}^{2}+e_{2}^{2}+e_{1} e_{2}\left(e_{1} e_{2}-4\right)\right)\right] \\
K_{23}=-K_{41}=-2 i \eta_{K} E I \beta_{1} \beta_{2}\left(r_{1}-r_{2}\right)\left(1-e_{1} e_{2}\right)\left(e_{1}-e_{2}\right) \\
K_{24}=K_{42}=-2 i \eta_{K} E I\left(r_{1}-r_{2}\right)\left[\beta_{1} e_{1}\left(1-e_{2}^{2}\right)+\beta_{2} e_{2}\left(e_{1}^{2}-1\right)\right] \\
K_{24}=-K_{43}=-i \eta_{K} E I\left[\left(\beta_{1}^{2} r_{2}+\beta_{2}^{2} r_{1}\right)\left(e_{1}^{2}+e_{2}^{2}-1-e_{1}^{2} e_{2}^{2}\right)+\beta_{1} \beta_{2}\left(r_{1}+r_{2}\right)\left(1+e_{1}^{2}+e_{2}^{2}+e_{1} e_{2}\left(e_{1} e_{2}-4\right)\right)\right] \\
1 \\
\text { and } \eta_{K}=\frac{1}{\left(\beta_{1}+\beta_{2}\right)^{2}\left(e_{1}^{2}+e_{2}^{2}\right)-\left(\beta_{1}-\beta_{2}\right)^{2}\left(1+e_{1}^{2} e_{2}^{2}\right)-8 \beta_{1} \beta_{2} e_{1} e_{2}}
\end{gathered}
$$




\section{REFERENCES}

[1] A. Tessler, M.D. Sciuva and M. Gherlone, Refinement of Timoshenko beam theory for composite and sandwich beams using zigzag kinematics, NASA, 2007.

[2] B. Arash and Q. Wang, A review on the application of nonlocal elastic models in modelling of carbon nanotubes and graphenes, Comput. Mater. Sci., Vol. 51, pp. 303-313, 2012. DOI: $10.1016 /$ j.commatsci.2011.07.040

[3] D.S. Kumar, D.R. Mahapatra and S. Gopalakrishnan, A spectral finite element for wave propagation and structural diagnostic analysis of composite beam with transverse crack, Finite Elements in Analysis and Design, Vol. 40, No. 13-14, pp. 1729-1751, 2004.

DOI: $10.1016 /$ i.finel.2004.01.001

[4] J.A. Franco-Villafane and R.A. Méndez-Sanchez, On the accuracy of the Timoshenko beam theory above the critical frequency: best shear coefficient, Journal of Mechanics, Vol. 32, No. 5, pp. 515-518, 2016. z-Sánchez (a1) DOI: 10.1017/jmech.2015.104

[5] G. Monsivais, A. Diaz-de-Anda, J. Flores, A. Morales and L. Gutiérrez, Experimental confirmation of the existence of the Timoshenko second spectrum, Proceeding of the $14^{\text {th }}$ Pan-American congress of Applied Mechanics, Santiago, Chile, 2014.

[6] L. Behera, S. Chakraverty, Free vibration of Euler and Timoshenko of nanobeams using boundary characteristic orthogonal polynomials, Applied Nanoscience, Vol. 4, No. 3, pp. 347-358, 2014. DOI: $10.1007 / \mathrm{s} 13204-013-0202-4$

[7] I. Senjanović, N. Vladimir and D.S. Chao, A shear locking-free beam finite element based on the modified Timoshenko beam, Transactions of FAMENA, Vol. 37, No. 4, pp. 1-16, 2014.

[8] M.A. El Shafei, FE modeling and analysis of isotropic and orthotropic beams using first order shear deformation theory, Materials Sciences and Application, Vol. 4, No. 1, pp. 77102, 2013. DOI: $\underline{10.4236 / \mathrm{msa} .2013 .41010}$

[9] A.J. Torrii and R.D. Machado, Structural dynamic analysis for time response of bars and trusses using the generalized finite element method, Latin American Journal of Solids and Structures, Vol. 1, pp. 1-13, 2012.

DOI: $\underline{10.1590 / \text { S1679-78252012000300001 }}$

[10] P. Lou, G.L. Dai and Q.J. Zeng, Dynamic analysis of a Timoshenko beam subjected to moving concentrated forces using the finite element method, Shock and Vibration, Vol. 14, No. 6, pp. 459-468, 2007. DOI: $10.1155 / 2007 / 460206$

[11] D.M. Yoshida and W. Weaver, Finite-element analysis of beams and plates with moving loads, Publ Int. Assoc. Bridge Struct. Eng., Vol. 31, No. 1, pp. 179-195, 1971.

DOI: $10.5169 /$ seals-24214

[12] J. Lee and W.W. Schultz, Eigenvalue analysis of Timoshenko beams and axisymmetric Mindlin plates by the pseudospectral method, Journal of Sound and Vibration, Vol. 269, pp. 609-621, 2004. DOI: $10.1016 /$ S0022-460X(03)00047-6

[13] S.S. Rao and R.S. Gupta, Finite element vibration analysis of rotating Timoshenko beams, Journal of Sound and Vibration, Vol. 242, No. 1, pp. 103-124, 2001.

DOI: $10.1006 /$ jsvi.2000.3362 
[14] R. Katz, C.W. Lee, A.G. Ulsoy and R.A. Scott, Dynamic response of a rotating shaft subject to a moving load, Journal of Sound and Vibration, Vol. 122, No. 1, pp. 131-148, 1988.

DOI: $10.1016 /$ S0022-460X(88)80011-7

[15] F.J. Doyle, Wave Propagation in Structures, Springer, New York, 1997.

[16] N. Azizi, M.M. Saadatpour and M. Mahzoon, Using spectral element method for analyzing continuous beams and bridges subjected to a moving load, Applied Mathematical Modelling, Vol.36, No. 8, pp. 3580-3592, 2012. DOI: 10.1016/i.apm.2011.10.019

[17] M. Krawczuk, M. Palacz and W. Ostachowicz, The dynamic analysis of a cracked Timoshenko beam by the spectral element method, Journal of Sound and Vibration, Vol. 264, No. 5, pp. 1139-1153, 2003. DOI:10.1016/S0022-460X(02)01387-1

[18] Y. Song and T.K. Usik, Vibration of a beam subjected to a moving force: Frequencydomain spectral element modeling and analysis, International Journal of Mechanical Sciences, Vol. 113, pp. 162-174, 2016. D0I: 10.1016/j.ijmecsci.2016.04.020

[19] M.G. Yawaraj and S. Rajneesh, A refined shear deformation theory of thick beams, Latin American Journal of Solids and Structures, Vol. 8, pp. 183-195, 2011.

DOI: $10.1590 /$ S1679-78252011000200005 